\title{
Requirement of future researches on burnout syndrome in interventional pain physicians in time of the COVID-19 pandemic
}

\author{
Sang Hun $\mathrm{Kim}^{1,2}$ \\ 'Department of Anesthesiology and Pain Medicine, School of Medicine, Chosun University, Gwangju, Korea \\ ${ }^{2}$ Department of Anesthesiology and Pain Medicine, Chosun University Hospital, Gwangju, Korea
}

Received March 8, 2021, Revised March 11, 2021, Accepted March 13, 2021

Handling Editor: Francis S. Nahm

Correspondence

Sang Hun Kim

Department of Anesthesiology and Pain Medicine, Chosun University Hospital, 365 Pilmun-daero, Dong-gu, Gwangju 61453, Korea

Tel: +82-62-220-3578, Fax: +82-62-223-2333, E-mail: ksh3223@chosun.ac.kr

The COVID-19 pandemic has intensified the workload of medical staff, causing them to suffer from burnout. Burnout syndrome occurring in medical personnel has been a commonly discussed issue in recent decades [1]. Some institutions are evaluating burnout for medical personnel, but there is still a lack of effort in removing or controlling the causes by institutions, or by the personnel themselves based on their individual experiences.

Among medical personnel involved in the pain management, burnout syndrome was more prevalent in comparison with primary care physicians [2]. Previous studies on burnout in pain physicians have reported its varying prevalence. Riquelme et al. [3] reported that $7.3 \%$ of pain physicians in Spain had burnout syndrome, with high levels of emotional exhaustion (22.6\%), high levels of depersonalization (22.3\%), and low levels of personal accomplishment (24.9\%) [3]. Ashton-James et al. [4] also reported that pain physicians in Australia showed high levels of emotional exhaustion (feeling drained or having "nothing left to give": $21.6 \%$ ), high levels of depersonalization (diminished concerns for patient outcomes: $14.2 \%$ ), and low levels of personal accomplishment (18.8\%). However, the prevalence of burnout syndrome showed differences between countries. Burnout syndrome among pain physicians revealed high levels of emotional exhaustion $(60.4 \%$ and $22.6 \%$, respectively), high levels of depersonalization (35.7\% and $22.3 \%$, respectively), and low levels of personal accomplishment (19.3\% and $24.9 \%$, respectively) in the United States and Spain [3,5].

Pain physicians can be emotionally burdened by pain management of patients with chronic pain, as well as negative emotions such as anxiety, frustration, disappointment, sadness, and anger [6]. Chronic pain patients often disclose private histories such as past trauma (emotional and physical) and life adversity [7]. Exposure to these patients' stories can generate empathy in pain physicians, and it can negatively affect their emotional and psychological conditions [4]. Hence, pain physicians have a higher risk of burnout syndrome with exposure to many chronic pain patients, who often present with high levels of emotional distress [4].

The higher level of burnout syndrome in pain physicians is usually associated with higher incidences of mental, physical, and social problems, including depression, (c) This is an open-access article distributed under the terms of the Creative Commons Attribution Non-Commercial License (http://creativecommons.org/licenses/by-nc/4.0/), which permits unrestricted non-commercial use, distribution, and reproduction in any medium, provided the original work is properly cited.

(c) The Korean Pain Society, 2021
Author contributions: Sang Hun Kim: Writing/manuscript preparation. 
suicidal ideation, poor communication of empathy/compassion, disease, alcohol/drug misuses, divorce, social withdrawal, relationship disruption, a lower success rate in interventions, and resignation $[4,8]$. So, burnout prevention and well-being promotion for pain physicians serve as an indispensable factor in improving clinical outcomes.

Burnout syndrome among pain physicians is known to be associated with low levels of experience, work overload, working alone, helplessness, and personal circumstances. So, the multidisciplinary pain clinic is useful in reducing the experience of burnout symptoms in comparison with private clinics where physicians are primarily working alone (high emotional exhaustion, $60.4 \%$; high depersonalization, $35.7 \%$ ) $[4,5]$. Many hospitals do not operate multidisciplinary pain clinics for pain management, and pain physicians are making decisions about pain management and treatment of patients completely autonomously. In these situations, they have limited opportunities to discuss complex cases, report after meeting emotionally challenged patients, or learn new pain management strategies from colleagues in other fields [4]. Therefore, the possibility of burnout for these pain physicians is higher.

Unlike overload, which is a known burnout risk factor, even a very low workload can cause burnout syndrome. Recently, the COVID-19 pandemic is leading to policies mandating enhanced physical distancing and minimizing personal contact, as well as restricting elective procedures and clinic visits, all of which impact the pain practice system. These policies have impacted most pain centers in hospital management, due to reduced patient visits and hospital income, as well as an increase in physicians' mental and physical fatigue, ultimately leading to burnout syndrome [1]. In some pain clinics, the effect of the COVID-19 pandemic has been unprecedented, stopping interventional pain procedures almost entirely, and causing significant financial, physical, and psychological stress. So, the COVID-19 pandemic contributed additional physical and emotional stress for interventional pain physicians, which may increase the risk of burnout syndrome [1].

In conclusion, burnout syndrome can be caused at any time by various factors such as physical, mental, and economic stress. Recognizing these problems, it is necessary to periodically conduct research and analysis of the degree of burnout for pain physicians, and efforts to reduce burnout should be provided effectively by the cooperation of governments, institutions, and individuals.

\section{CONFLICT OF INTEREST}

No potential conflict of interest relevant to this article was reported.

\section{FUNDING}

No funding to declare.

\section{ORCID}

Sang Hun Kim, https://orcid.org/0000-0003-3869-9470

\section{REFERENCES}

1. Jha SS, Shah S, Calderon MD, Soin A, Manchikanti L. The effect of COVID-19 on interventional pain management practices: a physician burnout survey. Pain Physician 2020; 23(4S): S271-82.

2. Sanfilippo F, Noto A, Foresta G, Santonocito C, Palumbo GJ, Arcadipane A, et al. Incidence and factors associated with burnout in anesthesiology: a systematic review. Biomed Res Int 2017; 2017: 8648925.

3. Riquelme I, Chacón JI, Gándara AV, Muro I, Traseira S, Monsalve V, et al. Prevalence of burnout among pain medicine physicians and its potential effect upon clinical outcomes in patients with oncologic pain or chronic pain of nononcologic origin. Pain Med 2018; 19: 2398-407.

4. Ashton-James CE, McNeilage AG, Avery NS, Robson LHE, Costa D. Prevalence and predictors of burnout symptoms in multidisciplinary pain clinics: a mixed-methods study. Pain 2021; 162: 503-13.

5. Kroll HR, Macaulay T, Jesse M. A preliminary survey examining predictors of burnout in pain medicine physicians in the United States. Pain Physician 2016; 19: E689-96.

6. Kennedy LC, Binswanger IA, Mueller SR, Levy C, Matlock DD, Calcaterra SL, et al. "Those conversations in my experience don't go well": a qualitative study of primary care provider experiences tapering long-term opioid medications. Pain Med 2018; 19: 2201-11.

7. Nelson S, Simons LE, Logan D. The incidence of adverse childhood experiences (ACEs) and their association with pain-related and psychosocial impairment in youth with chronic pain. Clin J Pain 2018; 34: 402-8.

8. Balch CM, Freischlag JA, Shanafelt TD. Stress and burnout among surgeons: understanding and managing the syndrome and avoiding the adverse consequences. Arch Surg 2009; 144: 371-6. 